Journal of the Egyptian Society of Parasitology, Vol.43, No.3, December 2013 J. Egypt. Soc. Parasitol., 43(3), 2013: 609 -616

\title{
UMBILICAL HERNIA IN CIRRHOTIC PATIENTS: OUTCOME OF ELECTIVE REPAIR
}

\section{By}

ADEL LASHEEN ${ }^{1}$, HATEM M. NASER ${ }^{2}$, AHMED ABOHASSAN ${ }^{3}$

Departments of General Surgery ${ }^{1}$, Anesthesia \& ICU ${ }^{2}$, and Internal Medicine $^{3}$, Faculty of Medicine, Al-Azhar University, Nasr City, Egypt

\begin{abstract}
Cirrhotic patients with umbilical hernia have an increased likelihood of complications following repair. The aim of this study was to assess the outcomes of elective umbilical hernia repair in cirrhotic patients. Fifty patients having uncomplicated umbilical hernia with a cirrhotic liver were studied prospectively. These patients divided into three groups' according to Child-Turcotte-Pugh (CTP) classification. After management of coagulopathy, correction of hypoalbuminaemia and electrolytes imbalance, and control of ascites, all patients underwent elective hernia repair under regional anesthesia. A comparison was made between the three groups as regard the size of the defect in the linea Alba, operative time, postoperative morbidity and mortality, length of hospital stay, time of return to daily life and postoperative changes in liver function tests $\left(\mathrm{LFT}_{\mathrm{S}}\right)$ in relation to the regional anesthesia applied. hernioplasty was done under spinal anesthesia in 13 patients $(26 \%)$, under epidural anesthesia in 10 patients $(20 \%)$, under intercostal nerve block in 7 patients (14\%), and under local anesthesia in 20 patients (40\%). There was an increased safety (less changes in $\mathrm{LFT}_{\mathrm{S}}$ ) in cases done under local anesthesia and intercostal nerve block. The overall complications rate was 30\%. There was an increased complications rate towards the decompensated cases. The differences in the mean length of hospital stay and mean time of return to daily life are statistically significant between the three groups. Umbilical hernia recurrence rate was $2 \%$ and no mortality was reported in the study groups.
\end{abstract}

Keywords: Umbilical hernia, cirrhotic liver, elective repair

\section{Introduction}

Umbilical herniation occurs in up to $20 \%$ of patients with advanced liver cirrhosis and ascites. Increased intraabdominal pressure as a result of ascites results in thrusting of the peritoneum forward through umbilical ring leading to herniation of the skin envelope above the abdominal wall (Tan and Chang, 2013).
The patients with advanced liver cirrhosis often have the protein-calorie malnutrition leading to abdominal wall muscle wasting, and this contributes to the increased incidence of umbilical hernia in these patients (Belghiti and Durand, 1997). Portosystemic collaterals may also result in recanalization of the umbilical vein and this contributes to further weakening of the ab- 
dominal wall and herniation of the umbilicus (Shlomovitz et al, 2005).

The most common complications of umbilical hernias in patients with cirrhosis and ascites include leakage, ulceration, rupture and incarceration. If such a complication is present, there is a high mortality rate after surgical repair. The elective repair is the most effective choice, as it prevents complications with a lower mortality. However, the control of ascites before and/or after repair is mandatory but may not always be possible with diuretics and paracentesis. The portal decompression by the transjugular intrahepatic portosystemic shunt (TIPS) with better control of ascites may allow these patients to undergo surgery. Patients with cirrhosis and umbilical hernias should be referred for elective surgical repair with mesh, preferably after optimal management of ascites (Triantos et al, 2011).

The management of umbilical hernias in cirrhotic patients with ascites is difficult as they often have advanced liver disease and at increased risk of complications following any surgical intervention. The elective surgical repair prevents complications, whereas mortality after emergency surgery is up to 30\% (Ammar, 2010). Optimal control of ascites before repair is essential and TIPS may be needed. If the liver transplantation is likely in the near future, repair can take place at the same time (Triantos et al, 2010).

The aim of this study was to assess the outcomes of elective umbilical hernia repair in the Egyptian cirrhotic patients.

\section{Patients, Subject and Methods}

This prospective study was carried out on fifty consecutive patients having umbilical hernia with a cirrhotic liver during the period from June 2011 till June 2013. All patients were admitted to departments of General Surgery, and Internal Medicine, Al-Hussein University Hospital and underwent elective hernia repair under regional anesthesia. Patients of both sexes and in different age groups were included.

For each patient beside usual routine preoperative examination, the detailed LFTS, bleeding profiles, serum electrolytes, hepatitis markers, abdominal ultrasonography, and the upper gastrointestinal endoscopy in some patients were done. All patients fulfilled the criteria of the American Society of Anesthesiologists (ASA) II and ASA III, and cirrhosis severity was determined by patient CTP classification.

These patients were divided into three groups; GI: included Child's A patients, GII: included Child's B patients, and GIII: included Child's C patients.

Inclusion criteria included the patients with noncomplicated and nonrecurrent umbilical hernia with cirrhotic liver. Exclusion criteria included complicated umbilical hernia (presented by strangulation, ulceration or rupture of overlying skin and leakage of ascites fluid), patients with hepatic focal lesions, Child's $\mathrm{C}$ patients with intractable ascites and patients with uncorrectable hypoalbuminaemia (serum albu$\min <24 \mathrm{~g} / \mathrm{L}$ ). Also, patients with uncorrectable comorbidities other than 
the comorbidities associated with the cirrhotic liver were excluded.

After management of coagulopathy, correction of the hypoalbuminaemia, and of electrolytes imbalance, control of ascites, and treatment of impending rupture oesophageal varices in some patients, all patients underwent elective hernia repair. Ascites was controlled by diuretics, infusion of human albumin, and paracentesis in some cases. The ordinary written informed consents were obtained in all patients, high risk consents were taken in some patients and umbilical hernia repair was done under regional anesthesia.

All patients were followed up postoperatively at two weeks, one month, three months, six months and nine months to detect any postoperative complications, assessment of wound healing, detection of recurrence and to detect any postoperative changes in the CTP classifications between groups.

A comparison was made between the three groups as regard the size of the defect in the linea Alba, operative time, postoperative morbidity and mortality, length of hospital stay, time of return to daily life and postoperative $\mathrm{LFT}_{\mathrm{S}}$ changes in relation to the regional anesthesia applied.

Statistical analysis: The data were computerized and analyzed by using SPSS. Comparisons between groups were tested using Chi-square test. A P value $<0.05$ was considered significant.

Anesthetic procedures: The spinal anesthesia was given by using the spinal needle 25 or 27 gauges in L4-L5 space, and $3 \mathrm{ml}$ heavy Bupivacaine was injected. Lumbar epidural anesthesia was used by injecting $15 \mathrm{ml}$ of Bupivacaine $0.5 \%$ in the epidural space. Two units of fresh frozen plasma (FFP) were given to the patients with spinal and epidural anesthesia to avoid hypotension during surgery. Intercostal nerve block; the safest approach for intercostal spaces is the mid-maxillary line with patient laying semi prone, using a needle 22 gauge. The needle connected to a syringe with interposition of an extension line, the needle was inserted just below the lower border of the upper rib at 80 degree angle to the chest pointing cephalic until contacts the rib. It is then slightly withdrawn and, redirected both more caudal and dorsally. A loss of resistance is felt at entry into the intercostal space. Advance the needle not more than $5 \mathrm{~mm}$ to prevent pneumothorax. Finally $2-3 \mathrm{~mL}$ of $0.5 \%$ Bupivacaine was injected at $9^{\text {th }}, 10^{\text {th }}$, and $11^{\text {th }}$ intercostal spaces on both sides after careful aspiration, as the intercostal artery and nerve are very close by. Local infiltration anesthesia by using 50:50 mixture of 2\% Lidocaine and $0.5 \%$ Bupivacaine with $1 / 100,000$ Epinephrine, an average of $30 \mathrm{ml}$ were used for infiltrations of abdominal wall layers around the umbilical hernia.

The operative technique: Through the transverse elliptical incision including the everted obliterated umbilicus with minimal removal of the redundant skin and minimal dissection in the subcutaneous plans, hernioplasty was done in all cases using outlay polypropylene mesh. The careful and individual liga- 
tions of the umbilical varices were done by Vicryle 2/0. The hernia sac and its contents had been dealt with, the peritoneum was closed separately and tightly by Vicryle $2 / 0$, the defect in the linea Alba was measured and repaired by continuous polypropylene sutures size 1 and reinforced by two to three interrupted sutures of the same suture material, and then a prosthetic polypropylene mesh was fixed. Suction drain was inserted in all patients after achievement of hemostasis. The subcutaneous tissues were closed by Vicryle $3 / 0$ and the skin by interrupted polypropylene $3 / 0$. Operative notes recorded including, type of regional anesthesia, operative time, size of the defect in the linea Alba, and intraoperative complications. Abdominal binders were applied in all patients. The drains were removed when a daily drainage below $20 \mathrm{~mL}$ was obtained. Skin sutures were removed alternatively on the 12 th and 15 th postoperative days.

\section{Results}

This study comprised of 50 patients and divided into three groups; group I: included 22 patients (12 males and 10 females) their age ranged from 40-58 years with mean age of $49.95 \pm 5.70$ years, group II: included 18 patients (11 males and 7 females) their age ranged from 44-62 years with mean age of $52.50 \pm 6.60$ years, and group III: included 10 patients $(6$ males and 4 females) their age ranged from 44-64 years with mean age of $54.40 \pm 6.60$ years. The differences in the mean age The differences in mean length of hospital stay and the mean time of return are statistically nonsignificant between the three groups (tab. 1).

Hernioplasty was done for patients under regional anesthesia. The regional anesthesia applied was; spinal in 13 patients $(26 \%)$, epidural in 10 patients $(20 \%)$, intercostal nerve block in seven patients $(14 \%)$ and local anesthesia in 20 patients $(40 \%)$. All operative procedures were successfully completed without any intraoperative complication related to surgery or anesthesia in all groups. Plain chest $\mathrm{x}$-ray (CXR) in erect position was done for patients who were subjected to hernioplasty under intercostal nerve block and compared with preoperative CXR that showed neither hemo nor pneumothorax. All were hemodynamically stable during the perioperative period, and no postoperative mortality occurred in any patients during the first postoperative month and during the follow up periods.

The mean size of the defect in the linea Alba was $2.54 \pm 0.43$ in GI, $2.75 \pm 0.66$ in GII, and $3.10 \pm 0.61$ in GIII. The mean operative time was $45.40 \pm 4.91$ in GI, $46.44 \pm 6.48$ in GII, and $46.90 \pm 7.86$ in GIII. Differences in the mean size of the defect in the linea Alba and the mean operative time are statistically not significant between the three groups. The mean length of hospital stay was $3.45 \pm 1.68$ in GI, $4.66 \pm$ 2.16 in GII and $19.00 \pm 8.49$ in GIII. The mean time to normal activity was $19.77 \pm 3.17$ days in GI, $25.94 \pm 5.82$ days GII, and $36.20 \pm 10.47$ in GIII. to normal activity were statistically significant. 
Postoperative complications (tab. 2) were reactionary hemorrhage in one patient $(2 \%)$ and stopped by the conservative measures in the form of repeated fresh blood transfusion, FFP and drugs used to control hemorrhage (Ethamsylate, Vit K1, and Tranexamic acid). Superficial wound sepsis occurred in 4 patients $(8 \%)$ and infections were treated successfully with proper antibiotics. Seroma formation occurred after removal of the suction drain in 2 patients (4\%) and treated by removing one suture and its evacuation. Partial wound dehiscence was occurred after removal of sutures in two patients (4\%) and these were healed after resuturing of the gapped wounds. Leakage of ascitic fluid was in one patient (2\%) and stopped spontaneously responding to diuretics. Encephalopathy occurred in one patient $(2 \%)$ and treated successfully in the general intensive care unit.

Among three patients with discomfort due to palpable prolene knots around the scar, one patient showed recurrence. The overall complications rate was $30 \%$. There was an increase in complications rate towards decompensated cases (i.e. Child's C cases) and the differences in the postoperative complications were significant between the three groups.

Postoperative changes in $\mathrm{LFT}_{\mathrm{S}}$ as regard type of regional anesthesia applied (compared to preoperative $\mathrm{LFT}_{\mathrm{S}}$ ): showed significant difference between GI and GII in all types of regional anesthesia and significant difference between GIII and other groups in local anesthesia. During the follow periods, one patient in GI (Child's A) was done under spinal anesthesia changed into Child's B, and three patients in GII (Child's B) changed into Child's C (two patients were done under spinal anesthesia and one patient was done under epidural anesthesia). The elevations in $\mathrm{LFT}_{\mathrm{S}}$ were resumed to the levels of preoperative ranges at the end of the $3^{\text {rd }}$ postoperative week except in one who developed encephalopathy and in patients who changed from Child's A into Child's B and patients who changed from Child's B into Child's C. There was an increased safety (less changes in $\mathrm{LFT}_{\mathrm{S}}$ ) in cases done under local anesthesia and intercostal nerve block (tab. 3). The mean follow up period was $6.27 \pm 2.25$ in GI, $6.00 \pm 2.30$ in GII, and 5.70 \pm 2.21 in GIII, and this difference was not significant (tab. 1).

Table 1: Demographic Mean \pm SD characteristics of patients under study

\begin{tabular}{|l|c|c|c|c|}
\hline Data & GI & GII & GIII & P Value \\
\hline Mean age & $49.95 \pm 5.70$ & $52.50 \pm 6.60$ & $54.40 \pm 6.60$ & 0.154 \\
\hline Size of defect in the linea Alba & $2.54 \pm 0.43$ & $2.66 \pm 0.66$ & $2.85 \pm 0.57$ & 0.102 \\
\hline Operative time & $45.40 \pm 4.91$ & $46.44 \pm 6.48$ & $46.90 \pm 7.86$ & 0.779 \\
\hline Mean length of hospital stay & $3.45 \pm 1.68$ & $4.66 \pm 2.16$ & $19.00 \pm 8.49$ & 0.000 \\
\hline Time of return to daily life & $19.77 \pm 3.17$ & $25.94 \pm 5.82$ & $36.20 \pm 10.47$ & 0.000 \\
\hline Follow up period & $6.27 \pm 2.25$ & $6.00 \pm 2.30$ & $5.70 \pm 2.21$ & 0.795 \\
\hline
\end{tabular}


Table 2: Postoperative complications in groups

\begin{tabular}{|c|c|c|c|c|}
\hline Complications & GI & GII & GIII & P Value \\
\hline Reactionary hemorrhage (2\%) & 0 & 0 & 1 & \multirow{10}{*}{0.000} \\
\hline Wound sepsis $(8 \%)$ & 1 & 1 & 2 & \\
\hline Seroma formation $(4 \%)$ & 0 & 1 & 1 & \\
\hline Wound dehiscence (4\%) & 0 & 1 & 1 & \\
\hline Leakage of ascitic fluid (2\%) & 0 & 0 & 1 & \\
\hline Encephalopathy (2\%) & 0 & 0 & 1 & \\
\hline Palpable prolene knots $(6 \%)$ & 1 & 1 & 1 & \\
\hline Recurrence $(2 \%)$ & 0 & 0 & 1 & \\
\hline Mortality $(0 \%)$ & 0 & 0 & 0 & \\
\hline TOTAL & $2 / 22(9.09 \%)$ & $4 / 18(22.22 \%)$ & $9 / 10(90 \%)$ & \\
\hline
\end{tabular}

Table 3: Postoperative changes in LFTS in relation to regional anesthesia applied

\begin{tabular}{|l|c|c|c|c|c|c|c|}
\hline \multirow{2}{*}{$\begin{array}{c}\text { Type of regional } \\
\text { anesthesia }\end{array}$} & \multicolumn{2}{c|}{ GI } & \multicolumn{2}{c|}{ GII } & \multicolumn{2}{c|}{$\begin{array}{c}\text { GIII } \\
\text { Value }\end{array}$} \\
\cline { 2 - 8 } & $\uparrow$ LFT $_{\mathrm{S}}$ & $\%$ & $\uparrow$ LFT $_{\mathrm{S}}$ & $\%$ & $\uparrow$ LFT $_{\mathrm{S}}$ & $\%$ & Valuen \\
\hline Spinal anesthesia (26\%) & $2 / 9$ & $22.2 \%$ & $3 / 4$ & $75 \%$ & - & - & 0.0026 \\
\hline Epidural anesthesia (20\%) & $1 / 7$ & $14.28 \%$ & $2 / 3$ & $66.66 \%$ & - & - & 0.0021 \\
\hline Intercostal nerve block (14\%) & $0 / 4$ & 0 & $1 / 3$ & $33.33 \%$ & - & $=$ & 0.0127 \\
\hline Local anesthesia (40\%) & $0 / 2$ & 0 & $2 / 8$ & $25 \%$ & $4 / 10$ & $40 \%$ & 0.008 \\
\hline
\end{tabular}

\section{Discussion}

Causes Cirrhosis of the Liver are fatty liver associated with obesity and diabetes, chronic viral infections of the liver (hepatitis B, C, \& D; Hepatitis D is extremely rare), blockage of the bile duct, which carries bile formed in the liver to the intestines, where it helps in the digestion of fats; in babies, this can be caused by biliary atresia in which the bile ducts are absent or damaged, causing bile to liver back up. In adults, bile ducts may become inflamed, blocked, or scarred, due to another liver disease called primary biliary cirrhosis, repeated bouts of heart failure with fluid backing up into liver and certain inherited diseases (Roziek et al, 2013).

The umbilical hernias in patients with cirrhosis are a difficult problem in high risk patients, and every surgeon has his or her own nightmare of a bad umbilical hernia case. Few surgeons want to fix these hernias, much less about their experience, such studies are still limited (Wong, 2012).

The patients with liver cirrhosis and umbilical hernia are often managed conservatively because of high postoperative mortality and morbidity with high postoperative recurrence (Tan and Chang, 2013). In patients whose ascites could be controlled by medical therapy, surgical herniorrhaphy was performed safely with low mortality and morbidity (Belghiti and Durand, 1997).

For many years, surgical dogma stipulated repair of umbilical hernia only in patients who developed complications. Conservative management of umbilical hernias in patients with liver cirrhosis and ascites led to a high rate of incarcerations with subsequent hernia repair in an emergency setting, whereas elective repair could be done with less morbidity and was therefore advocated (Perkins, 2006). 
Until the 1980s, the standard practice had been to forego the elective repair of umbilical hernias in patients with ascites because of fear of high morbidity and mortality rates, and a high incidence of postoperative hernia recurrence. However, review of the literature documents that the mortality rate for elective repair of umbilical hernias in patients with ascites is only $2 \%$ compared with, a $14 \%$ mortality rate for emergent repair. Therefore if a patient can be adequately prepared for surgery, elective repair is indicated (Fagan et al, 2004).

Hernia operation could be done safely in CTP class A and B with low rate of recurrences, and there was no definitive increase in the operative risk in class C. Also, refractory ascites did not increase operative risk and recurrence rate. Thus, surgical repair could be recommended even in patients with refractory ascites and poor hepatic function to prevent life-threatening complications or severe pain (Park et al, 2007).

In the series reported by the French Association of Surgery which included 81 patients who underwent surgical treatment for umbilical hernia, overall mortality was $5 \%$ : $11 \%$ after the emergency surgery for ruptured or strangulated umbilical hernia and 2\% after elective surgery (Gillet et al, 1993).

The mortality was zero in two studies by the expert centers, including the 39 and 40 patients respectively that underwent umbilical hernia surgery (Leonetti et al, 1984).

In the present study, the elective hernioplasty was done under spinal anesthesia in 13 patients $(26 \%)$, under epidural anesthesia in 10 patients $(20 \%)$, under intercostal nerve block in seven patients $(14 \%)$, and under local anesthesia in 20 ones $(40 \%)$. All the operative procedures were successfully done with no intraoperative complications were related neither to surgery nor to anesthesia. There was an increased safety (less changes in $\mathrm{LFT}_{\mathrm{S}}$ ) in cases done under local anesthesia and intercostal nerve block. The overall complications rate was $30 \%$. There was increased complications rate towards the decompensated cases (i.e. Child's C cases).

The differences in postoperative complications showed significance between the three groups, and there was statistically significant difference in the mean length of hospital stay and the mean time of return to daily life among the three groups. The umbilical hernia recurrence rate was $2 \%$ and no mortality was reported in the study groups. Postoperative changes in CTP classification may be due to hypotension during surgery. These agreed with results of Belghiti et al. (1990).

\section{Conclusion}

Generally speaking, the rate of liver cirrhosis and its complications is increasing worldwide including Egypt

The elective repair of umbilical hernia in cirrhotic patients under the regional anesthesia was associated with low morbidity and mortality when the comorbidities associated with cirrhosis were medically corrected and should be advocated to avoid life-threatening 
complication associated with conservative management.

\section{References}

Ammar, SA, 2010: Management of complicated umbilical hernias in cirrhotic patients using permanent mesh: randomized clinical trial. Hernia 14:353.

Belghiti, J, Durand, F, 1997: Abdominal wall hernias in the setting of cirrhosis. Seminars in Liver Disease17, 3: 219-26.

Belghiti. J, Desgrandchamps, F, Farges, O, Fékété, F, 1990: Herniorrhaphy and concomitant peritoneovenous shunting in cirrhotic patients with umbilical hernia. Wld. J. Surg. 14: 242-6.

Fagan, SP, Awad, SS, Berger, DH, 2004: Management of complicated umbilical hernias in patients with endstage liver disease and refractory ascites. Surgery 135:679-82.

Gillet, M, Chirurgie de la Paroi chez le, 1993: Cirrhotique In: Belghiti J, Gillet M Editors La chirugie digestive chez le cirrhotique. Paris: Monographies de l'AFC, pp. 53-60

Leonetti, JP, Aranha, GV, Wilkinson, W A, Stanley, M, Greenlee, HB, 1984: Umbilical herniorrhaphy in cirrhotic patients. Arch Surg. 119:442-5.

Park, JK, Lee, SH, Yoon, WJ, Lee, J $\mathrm{K}$, Park, SC, et al, 2007: Evaluation of hernia repair operation in Child-Turcotte-Pugh class $\mathrm{C}$ cirrhosis and refractory ascites J. Gastroenterol. Hepatol. 22, 3:377-82.

Perkins, JD, 2006: Liver Transplantation Worldwide, University of Washington Medical Center, Seattle, WA Volume 14, Issue 1, USA, pp. 110-14.
Roziek, MS, Hammam, OA, Abd ElSattar, H, El-Tiby, DM, El-Dessouky, YM, 2013: Expression of Fas protein (CD95) and Fas ligand in liver tissue of patients with $\mathrm{HCV}$-induced chronic liver disease and its correlation with the disease progression. J. Egypt. Soc. Parasitol. 43, 1:235-44.

Shlomovitz, E, Quan, D, Etemad-Rezai, R, McAlister, VC, 2005: Association of recanalization of the left umbilical vein with umbilical hernia in patients with liver disease. Liver Transplantation 11, 10:1298-99.

Tan, HK, Chang, PE, 2013: Acute Abdomen Secondary to Incarcerated Umbilical Hernia after Treatment of Massive Cirrhotic Ascites. Case Reports in Hepatology; Article ID948172, 3 pages http://dx.doi.org/10.1155/

Triantos, CK, Kehagias, I, Nikolopoulou, V, Burroughs, AK, 2010: Incarcerated umbilical hernia after large volume paracentesis for refractory ascites. J. Gastrointest. Liver Dis. 19, 3: 245-52

Triantos, CK, Kehagias, I, Nikolopoulou, V, Burroughs, AK, 2011: Surgical repair of umbilical hernias in cirrhosis with ascites. Am. J. Med. Sci. 341, 3:222-6.

Wong, LL, 2012: Should a patient with cirrhosis have an Innie or an Outie? Arch. Surg. 147, 9:869-70. 